\title{
Survival of Developing Motor Neurons Mediated by Rho GTPase Signaling Pathway through Rho-Kinase
}

\author{
Kenta Kobayashi, ${ }^{1}$ Masanori Takahashi, ${ }^{2}$ Natsuki Matsushita, ${ }^{3}$ Jun-ichi Miyazaki, ${ }^{4}$ Masato Koike, ${ }^{5}$ \\ Hiroyuki Yaginuma, ${ }^{5}$ Noriko Osumi, ${ }^{2}$ Kozo Kaibuchi, ${ }^{6}$ and Kazuto Kobayashi ${ }^{1}$ \\ ${ }^{1}$ Department of Molecular Genetics, Institute of Biomedical Sciences, Fukushima Medical University School of Medicine, Fukushima 960-1295, Japan, \\ ${ }^{2}$ Department of Developmental Neurobiology, Tohoku University Graduate School of Medicine, Sendai 980-8575, Japan, ${ }^{3}$ Department of Embryology, \\ Institute for Developmental Research, Aichi Human Service Center, Kasugai 480-0392, Japan, ${ }^{4}$ Division of Stem Cell Regulation Research, Osaka University \\ Graduate School of Medicine, Suita 565-0871, Japan, ${ }^{5}$ Department of Anatomy, Fukushima Medical University School of Medicine, Fukushima 960-1295, \\ Japan, and ${ }^{6}$ Department of Cell Pharmacology, Nagoya University Graduate School of Medicine, Nagoya 466-8550, Japan
}

A variety of neurons generated during embryonic development survive or undergo programmed cell death (PCD) at later developmental stages. Survival or death of developing neurons is generally considered to depend on trophic support from various target tissues. The small GTPase Rho regulates diverse cellular processes such as cell morphology, cell adhesion, cell motility, and apoptosis. Rho-dependent serine-threonine protein kinase (Rho-kinase-ROK-ROCK), one of the effector proteins, transmits signals for some Rho-mediated processes. Here, we report the in vivo role of the Rho signaling pathway through Rho-kinase during development of motor neurons (MNs) in the spinal cord. We performed conditional expression of a dominant-negative form for RhoA (RhoA DN) or for Rho-kinase (Rho-K DN) in transgenic mice by using the Cre-loxP system to suppress the activity of these signaling molecules in developing MNs. Expression of RhoA DN reduced the number of MNs in the spinal cord because of increased apoptosis while preserving the gross patterning of motor axons. Expression of Rho-K DN produced developmental defects similar to those observed in RhoA DN expression. In addition, analysis of transgenic mice expressing Rho-K DN showed that the increased apoptosis of MNs was induced at the early embryonic stages before the initiation of PCD, and that MN death at the late embryonic stages corresponding to the period of PCD was moderately enhanced in the transgenic mice. These findings indicate that the Rho signaling pathway, primarily through Rho-kinase, plays a crucial role in survival of spinal MNs during embryogenesis, particularly at the early developmental stages.

Key words: small GTPase signaling; neuronal survival; programmed cell death; apoptosis; axon patterning; motor neuron

\section{Introduction}

A variety of neurons generated during embryonic development survive or undergo programmed cell death (PCD) at later developmental stages. A general mechanism that controls survival or death of developing neurons is considered to depend on trophic support derived from various sources including their target tissues (Oppenheim, 1991). In particular, a variety of neurotrophic factors support neuronal survival through specific intracellular signaling pathways (Pettmann and Henderson, 1998; Sendtner et al., 2000; Huang and Reichardt, 2001). In contrast, recent evi-

\footnotetext{
Received July 29, 2003; revised Feb. 20, 2004; accepted Feb. 21, 2004.

This work was supported by grants-in-aid from the Ministry of Education, Science, Culture, and Sports of Japan, by Core Research for Evolutional Science and Technology, and by Solution Oriented Research for Science and Technology of the Japan Science and Technology Corporation. We thank Dr. W. Müller for providing the Cre recombinase gene, Dr. F. Murakami for critical reading of this manuscript, Drs. J. Takeda and M. Amano for valuable discussion, Dr. H. Shimada and I. Takahashi for immunohistochemistry, S. Nabeshima-Matsushita for histological detection of $\beta$-gal activity, and K. Takahashi for generation of transgenic mice.

Correspondence should be addressed to Dr. Kazuto Kobayashi, Department of Molecular Genetics, Institute of Biomedical Sciences, Fukushima Medical University School of Medicine, Fukushima 960-1295, Japan. E-mail: kazuto@fmu.ac.jp.

M. Koike's present address: Department of Cell Biology and Neuroscience, Osaka University Graduate School of Medicine, A1, 0saka 565-0871, Japan.

DOI:10.1523/JNEUROSCI.0295-04.2004

Copyright $\odot 2004$ Society for Neuroscience $\quad 0270-6474 / 04 / 243480-09 \$ 15.00 / 0$
}

dence suggests the presence of a mechanism that regulates neuronal survival or death independently of target-derived trophic support (Oppenheim et al., 2001). For instance, some neuronal populations in chick embryo undergo PCD before the establishment of synaptic connections at early embryonic stages (Homma et al., 1994; Yaginuma et al., 1996). In primary cultures, embryonic neurons at certain developmental stages survive independently of exogenous trophic support (Mettling et al., 1995).

The small GTPase Rho acts as an intracellular molecular switch that transduces extracellular signals to the cytoskeleton and the nucleus, and it exists as a subfamily comprising RhoA, RhoB, and RhoC isoforms (Hall, 1998; Kaibuchi et al., 1999). The signaling through Rho GTPase is known to mediate the regulation of diverse cellular processes, including cell morphology, cell adhesion, cell motility, and apoptosis (Kaibuchi et al., 1999; Aznar and Lacal, 2001). Especially, such signaling mediates growth cone behavior and dendritic morphogenesis of neurons (Luo, 2000). Rho-dependent serine-threonine protein kinase (Rhokinase-ROK-ROCK) is one of the Rho effector proteins and exists as two isoforms, $\mathrm{ROK} \alpha / \mathrm{ROCKII}$ and $\mathrm{ROK} \beta / \mathrm{ROCKI}$ (Leung et al., 1995; Ishizaki et al., 1996; Matsui et al., 1996). Rho-kinase transmits signals for some cellular processes downstream of Rho (Amano et al., 1997, 1998; Hirose et al., 1998). Genetic studies in 
A

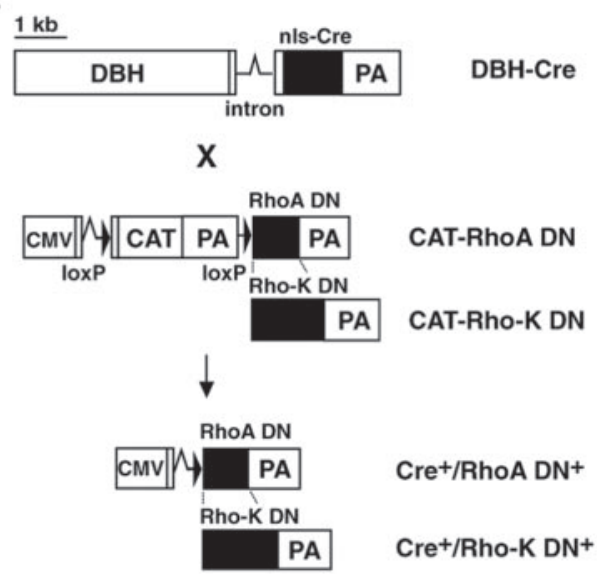

C

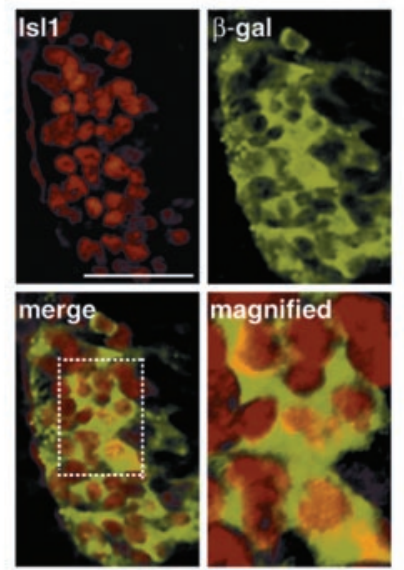

B

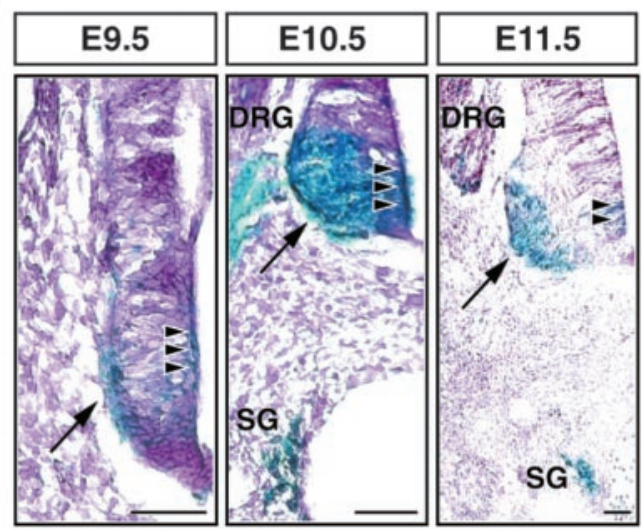

D

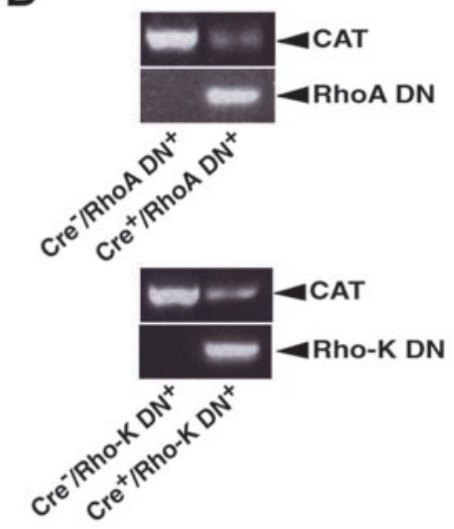

Figure 1. Expression of RhoA DN and Rho-K DN by using the Cre-loxP-mediated recombination system. $A$, Experimental strategy. DBH-Cre transgenic mice were crossed with CAT-RhoA DN or CAT-Rho-K DN mice. In the double-transgenic mice ( $\mathrm{Cre}^{+} /$ $\mathrm{RhoA} \mathrm{DN}^{+}$or $\mathrm{Cre}^{+} / \mathrm{Rho}^{-\mathrm{K} \mathrm{DN}^{+}}{ }^{+}$), (re-loxP recombination deletes the (AT gene cassette in specific cell types, thereby leading to the expression of the dominant-negative forms. $B$, Patterns of Cre-loxP recombination. Histological staining of $\beta$-gal activity in transverse sections through the thoracic spinal cord of transgenic embryos carrying both DBH-Cre and CAG-CAT-Z transgenes $\left(\mathrm{Cre}^{+} / \mathrm{lacZ}^{+}\right)$at E9.5-E11.5, showing the staining signals in the developing MN columns in the ventrolateral region (arrow) and in the presumptive progenitor cells in the region around the ventricular zone (arrowheads) of the neural tube. The signals were also observed in the SG and DRG. Scale bar, $50 \mu \mathrm{m}$. C, Cre-loxP recombination in developing MNs. Sections through the thoracic spinal cord of the $\mathrm{Cre}^{+} / / \mathrm{acZ}{ }^{+}$double-transgenic embryos at E10.5 were stained with antibodies for IsI1 and $\beta$-gal. Isl1-staining signal (red), $\beta$-gal-staining signal (green), and their merged image are indicated. As seen in a fourfold-magnified view of the merged image, Is 11 and $\beta$-gal signals are localized in the nucleus and the soma, respectively. Thus, double-positive cells show a red nucleus and a green soma in a single cell. Scale bar, $50 \mu \mathrm{m}$. D, Expression of RhoA DN and Rho-KDN genes. Total RNA prepared from E10.5 embryos was reverse transcribed and analyzed by PCR amplification with CAT, RhoA DN, or Rho-K DN primer set. The size of amplified DNA was 390 bp for CAT, 617 bp for RhoA DN, and 482 bp for Rho-K DN.

Drosophila have indicated the in vivo functions of Rho or Rhokinase in gastulation, segmentation, and generation of tissue polarity during embryonic development (Barrett et al., 1997; Strutt et al., 1997; Magie et al., 1999; Winter et al., 2001). In addition, recent studies have shown the role of these signaling molecules in vertebrate embryonic morphogenesis (Wünnenberg-Stapleton et al., 1999; Wei et al., 2001).

The development of motor neurons (MNs) provides a useful model system for investigation of the molecular mechanisms underlying specification of neuronal fate, axon patterning, and neuronal survival in the vertebrate CNS. In the present study, we addressed the in vivo role of the Rho/Rho-kinase signaling pathway during development of MNs in the spinal cord. We performed conditional expression of a dominant-negative form for RhoA (RhoA DN) or Rho-kinase (Rho-K DN) in MNs in transgenic mice to suppress the activity of all isoforms of Rho or Rho- kinase. We used this transgenic strategy to rule out the possibility that phenotypic effects by knock-out of the gene encoding a given isoform may be masked by redundant functions of other isoforms. Our data show that the Rho signaling pathway, primarily through Rho-kinase, plays an essential role in the survival of the spinal MNs during mammalian embryogenesis.

\section{Materials and Methods}

Construction of transgenes. A $1.2 \mathrm{~kb}$ DNA fragment encoding Cre recombinase with a nuclear location signal was inserted into the EcoRI site of the pBST-N plasmid (Kobayashi et al., 1992). The $4 \mathrm{~kb} 5^{\prime}$-flanking region of human dopamine $\beta$-hydroxylase (DBH) gene (Kobayashi et al., 1992) was inserted upstream of the resultant plasmid to generate $\mathrm{pDBH}$-Cre. A $0.9 \mathrm{~kb}$ DNA fragment containing the cytomegalovirus (CMV) early gene promoter was fused to the rabbit $\beta$-globin second intron and chloramphenicol acetyltransferase (CAT) gene cassette connected to a polyadenylation signal (PA) (Sasaoka et al., 1992), both ends of which were flanked by loxP sites. Another polyadenylation signal was connected downstream of the second loxP site, making a unique $\mathrm{ClaI}$ site between the loxP site and the $5^{\prime}$-end of the polyadenylation signal to generate pCMV-CAT-PA. A $0.6 \mathrm{~kb}$ DNA fragment encoding a dominant-negative mutant for RhoA or RhoA ${ }^{\mathrm{N} 19}$ (Amano et al., 1998), termed RhoA DN, or a 1.4 kb DNA fragment encoding a dominant-negative mutant for Rho-kinase or $\mathrm{RB} / \mathrm{PH}(\mathrm{TT})$ (Amano et al., 1998), termed Rho-K DN, was inserted into the ClaI site of pCMV-CAT-PA, resulting in pCAT-RhoA DN or pCAT-Rho-K DN.

Generation of transgenic mice. The transgene was microinjected into fertilized mouse eggs, which were then implanted in pseudopregnant females. Transgenic mice were identified by Southern blot hybridization with genomic DNA prepared from tail clips. The DBH-Cre transgenic mice were crossed with reporter mice carrying the pCAG-CAT-Z fusion gene containing a chicken $\beta$-actin gene promoterloxP-CAT gene-loxP- $\beta$-galactosidase $(\beta$-gal) gene (Araki et al., 1995). The double-transgenic embryos carrying both DBH-Cre and CAGCAT- $Z$ transgenes were analyzed for Cre-loxPmediated recombination by histological detection of $\beta$-gal activity. In this study, we used the transgenic line termed DBH-Cre/6-8, which exhibited an efficient recombination in developing MNs (see below). For the CAT-RhoA DN or CAT-Rho-K DN transgene, the transgenic lines obtained were analyzed for expression of CAT mRNA in the spinal cord by Northern blot hybridization. In this study, we used two lines designated CAT-RhoA DN/4-18 and CAT-Rho-K DN/3-1, both of which expressed high levels of CAT mRNA in their tissue. There was no developmental defect in DBH-Cre/6-8, CAT-RhoA DN/4-18, or CATRho-K DN/3-1 transgenic mice. All mice were handled according to the guidelines established by the Fukushima Medical University animal facility.

Genotyping of embryos. Transgenes were identified by PCR analysis of genomic DNA prepared from the yolk sac. The primers used were $5^{\prime}$ ACCAGCCAGCTATCAACTCG-3' and 5'-TTACATTGGTCCAGCCACC-3' for DBH-Cre, 5'-GCGTTACCCAACTTAATCG-3' and 5'TGTGAGCGAGTAACAACC-3' for CAG-CAT-Z, 5' ${ }^{\prime}$-ACTCATCT- 
CAGAAGAGGATCTG-3' and 5'-TCACAAGACAAGGCAACCAGATT-3' for CAT-RhoA DN, and 5'-ACTCATCTCAGAAGAGGATCTG-3' and 5'TTCATTCAGTTCTTTCTGATATTTG-3' for CAT-Rho-K DN.

Histological detection of $\beta$-gal activity. Embryos were dissected out at various developmental stages and fixed in $4 \%$ paraformaldehyde solution. Transgenic embryos doubly carrying the DBH-Cre and CAGCAT-Z transgenes were transversely cut into sections $(20 \mu \mathrm{m})$ with a cryostat. The sections were stained in a solution containing $1 \mathrm{mg} / \mathrm{ml}$ 4-chloro-5-bromo-3-indolyl- $\beta$-galactoside, $5 \mathrm{~mm} \mathrm{~K}_{3} \mathrm{Fe}(\mathrm{CN})_{6}, 5 \mathrm{~mm}$ $\mathrm{K}_{4} \mathrm{Fe}(\mathrm{CN})_{6}, 2 \mathrm{~mm} \mathrm{MgCl}, 0.01 \%$ sodium deoxycholate, and $0.02 \%$ Nonidet P-40 in PBS. They were then counterstained with neutral red.

Immunohistochemistry and imaging. Embryos were fixed and processed for immunohistochemistry as described previously (Takahashi and Osumi, 2002). Transverse sections $(10 \mu \mathrm{m})$ were incubated with mouse monoclonal antibodies (Abs) against Isl1 (39.4D6; Developmental Studies Hybridoma Bank, Iowa City, IA; 1:100) and neurofilament (2H3; Developmental Studies Hybridoma Bank; 1:100), rabbit polyclonal Abs against $\beta$-gal (Cappel, West Chester, PA; 1:300), myosinbinding subunit (MBS) of myosin phosphatase (Kawano et al., 1999) (1:100), MBS phosphorylated at Thr-850 (Upstate Biotechnology, Lake Placid, NY; 1:100), S100 (Dako, High Wycombe, UK, Glostrup, Denmark; 1:200), and rabbit monoclonal $\mathrm{Ab}$ against an active form of caspase-3 (PharMingen, San Diego, CA; 1:200). FITC-conjugated goat anti-rabbit IgG, cyanin 3 (Cy3)-conjugated goat anti-rabbit IgG, and Cy3-conjugated donkey anti-mouse IgG (Jackson ImmunoResearch, West Groove, PA; 1:500) were used as secondary Abs. Fluorescence images for tissue sections were obtained under a Leica (Nussloch, Germany) microscope (DMRE HC) and a Zeiss (Thornwood, NY) confocal laser microscope (LSM510) equipped with proper filter cube specifications for FITC and Cy3 fluorescence channels. The images were acquired with a high-grade CCD camera system controlled by a Zeiss Axiovision software package.

Quantification of the intensity of fluorescence in single cells was performed as described previously (Matsushita et al., 2002). Fluorescence images obtained from double immunostaining with anti-Isll $\mathrm{Ab}$ and either anti-MBS or antiphosphorylated MBS Ab were obtained under a constant exposure condition, in which the intensity in a given pixel did not become saturated. The fluorescence intensity of MBS and phosphorylated MBS signals in Isl1-positive cells was measured by Simple PCI software (Complix, Cranberry Township, PA).

Reverse transcription (RT)-PCR. Embryos were homogenized in ISOGEN (Nippon Gene, Toyama, Japan) for total RNA extraction. The RNA samples $(1 \mu \mathrm{g})$ were reverse transcribed by using Molony murine leukemia virus reverse transcriptase (Promega, Madison, WI), and the resultant cDNA was amplified by PCR. The primers used for CAT detection were $5^{\prime}$-CAGTCAGTTGCTCAATGTACC-3' and 5' -ACTGGTGAAACTCACCCA- $3^{\prime}$. The primers used for RhoA DN and Rho-K DN were described above in Genotyping of Embryos.

Terminal deoxynucleotidyl transferase-mediated dUTP-biotin nick end labeling. Terminal deoxynucleotidyl transferase-mediated biotinylated UTP nick end labeling (TUNEL) staining was performed as described previously (Takahashi and Osumi, 2002) with minor modification. Sections $(10 \mu \mathrm{m})$ were incubated in $150 \mathrm{~mm}$ sodium cacodylate buffer, $\mathrm{pH}$ 6.6, containing $1.5 \mathrm{~mm} \mathrm{CoCl}_{2}, 500 \mathrm{~nm}$ biotin-14 dATP, and $120 \mathrm{U} / \mathrm{ml}$ terminal deoxynucleotidyl transferase (Roche Products, Mannheim, Germany). The incorporated biotin-14 dATP was detected with streptoavidin-Oregon Green 488 conjugates (Molecular Probes, Eugene, OR).

Axonal labeling. DiI or 1,1'-diocadecyl-3,3,3',3'-tetramethylindocarbocyanine perchlorate (Molecular Probes) was dissolved at $0.1 \%$ in a mixture of ethanol and dimethylsulfoxide $(9: 1)$ and pressure-injected into the ventral region of the brachial spinal cord, limb, or myotome (Yaginuma et al., 1996). Three days after the injection, transverse sections $(150 \mu \mathrm{m})$ were cut with a vibratome and observed under a Leica microscope.

Electron microscopy. Embryos were fixed and processed for electron microscopy as described previously (Yaginuma et al., 1996). After they were washed thoroughly with $0.1 \mathrm{M}$ phosphate buffer containing $7.5 \%$ sucrose, $\mathrm{pH}$ 7.4, the samples were postfixed with $1 \% \mathrm{OsO}_{4}$ in the same
Table 1. Quantification of the phosphorylation level of MBS

\begin{tabular}{lll}
\hline & \multicolumn{2}{l}{ Relative fluorescence intensity (cell number) } \\
\cline { 2 - 3 } Genotype & MBS & Phosphorylated MBS \\
\hline RhoA DN & & \\
$\quad$ Wild type & $1.00 \pm 0.05(525)$ & $1.00 \pm 0.01(466)$ \\
$\quad$ Cre $/$ RhoA DN $^{+}$ & $0.98 \pm 0.05(480)$ & $0.69 \pm 0.03^{* *}(472)$ \\
Rho-K DN & & \\
$\quad$ Wild type & $1.00 \pm 0.05(486)$ & $1.00 \pm 0.01(476)$ \\
Cre $^{+} /$Rho-K DN $^{+}$ & $1.00 \pm 0.07(511)$ & $0.79 \pm 0.07^{*}(487)$ \\
\hline
\end{tabular}

The sections through the thoracic spinal cord of the wild-type and double-transgenic embryos at E10.5 were stained with anti-|s $11 \mathrm{Ab}$ and either anti-MBS or antiphosphorylated MBS Ab. The fluorescence intensity of MBS or phosphorylated MBS signals in single IsI1-positive cells was measured, and the relative fluorescence intensity was calculated as the ratio of the fluorescence intensity relative to the average of the intensity in the wild-type embryos. Data are the mean \pm SEM ( $n=4$ for each genotype). The number of cells used for measurement of the fluorescence intensity is shown in parentheses. Asterisks indicate $p$ values according to Student's $t$ test; ${ }^{*} p<0.01,{ }^{* *} p<0.001$ versus wild-type mice.

buffer at $4^{\circ} \mathrm{C}$ for $2 \mathrm{hr}$ and block-stained with a $2 \%$ aqueous solution of uranyl acetate for $1 \mathrm{hr}$. The tissues were then dehydrated by passage through a graded series of ethanol and embedded in Epon 812 (TAAB, Berks, UK). Thin sections $(0.1 \mu \mathrm{m})$ were cut on an ultramicrotome, stained with uranyl acetate and lead citrate, and examined under a JEM1200 EX electron microscope (Jeol, Tokyo, Japan).

\section{Results}

\section{Conditional expression of RhoA DN and Rho-K DN in transgenic mice}

To suppress the activity of Rho and Rho-kinase during MN development, we aimed to express RhoA DN or Rho-K DN in specific neuronal types by using the Cre-loxP-mediated recombination system (Gu et al., 1993) (Fig. 1A). We generated DBH-Cre transgenic mice that express Cre recombinase under the control of the $4 \mathrm{~kb}$ upstream sequence of the human DBH gene, which is known to drive the expression of the gene in some neuronal types such as the sympathetic, motor, and sensory neurons (Kapur et al., 1991; Mercer et al., 1991; Kobayashi et al., 1992). To characterize the Cre-loxP recombination, we crossed the DBH-Cre transgenic mice with reporter mice carrying the pCAG-CAT-Z transgene and analyzed the double-transgenic embryos carrying two kinds of transgenes $\left(\mathrm{Cre}^{+} / \mathrm{lac}^{+}\right)$by histological staining for $\beta$-gal activity (Fig. $1 B$ ). At embryonic day (E) 9.5, the staining signals were visualized in developing $\mathrm{MNs}$ in the ventral region of the neural tube. The signals were also localized in their presumptive progenitor cells in a region around the ventricular zone. At E10.5 and E11.5, the signals were visualized in the MN columns in addition to being detected in the sympathetic ganglion (SG) and in part of the dorsal root ganglion (DRG). The sections of the $\mathrm{Cre}^{+} / \mathrm{lacZ}^{+}$embryos at E10.5 were subsequently stained with anti-Isll and anti- $\beta$-gal Abs. The $\beta$-gal expression was found in the majority of Isl1-positive neurons $(90-95 \% ; n=4)$ (Fig. 1C). These results indicate that Cre-loxP recombination occurred in the developing MNs of the transgenic embryos depending on the specificity of the $\mathrm{DBH}$ gene promoter.

Next, we generated the transgenic mice (CAT-RhoA DN or CAT-Rho-K DN) that carry the CMV gene promoter fused to the CAT gene cassette flanked by two loxP sites and either the RhoA DN or Rho-K DN gene cassette (Fig. $1 A$ ). These mice were mated with the $\mathrm{DBH}$-Cre mice to obtain double-transgenic mice $\left(\mathrm{Cre}^{+} /\right.$ $\mathrm{RhoA} \mathrm{DN}^{+}$or Cre $\left.{ }^{+} / \mathrm{Rho}_{\mathrm{K} \mathrm{DN}}{ }^{+}\right)$. To assess the expression of the dominant-negative forms by Cre-loxP recombination, we performed RT-PCR analysis on $\mathrm{Cre}^{+} / \mathrm{RhoA} \mathrm{DN}^{+}$and Cre ${ }^{+} /$ Rho-K DN ${ }^{+}$embryos at E10.5 (Fig. $1 D$ ). The transcripts of RhoA $\mathrm{DN}$ or Rho-K DN were detected in the corresponding doubletransgenic embryos but not in either the $\mathrm{Cre}^{-} / \mathrm{RhoA} \mathrm{DN}^{+}$or the 


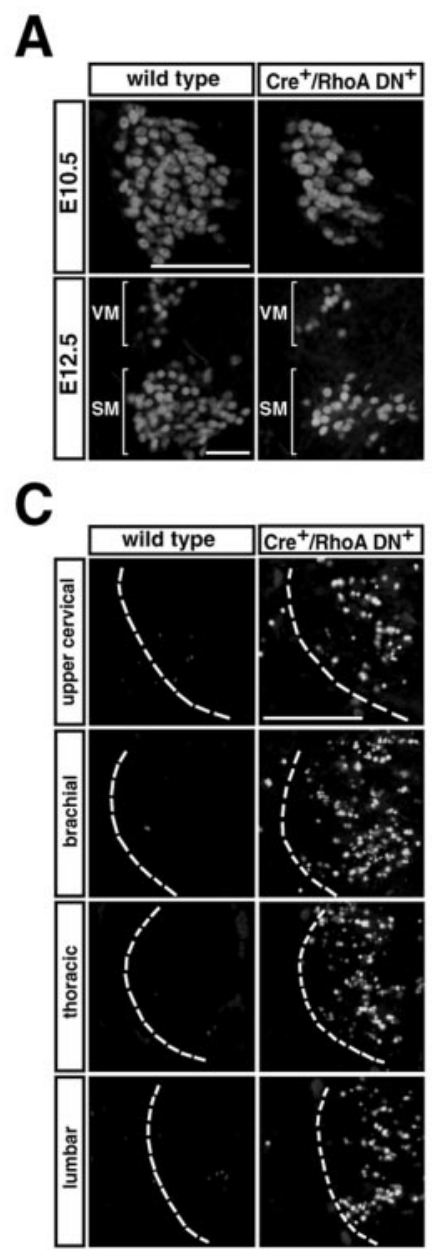

\section{B}

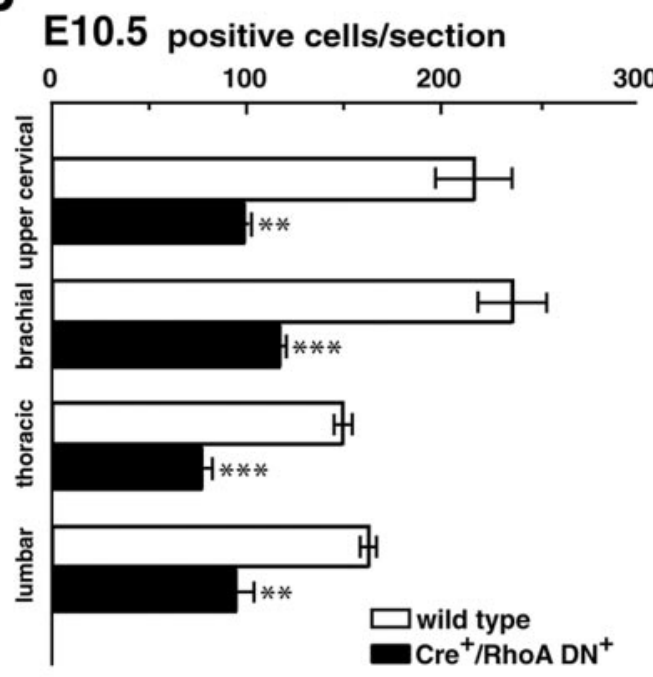

\section{E12.5 positive cells/section}

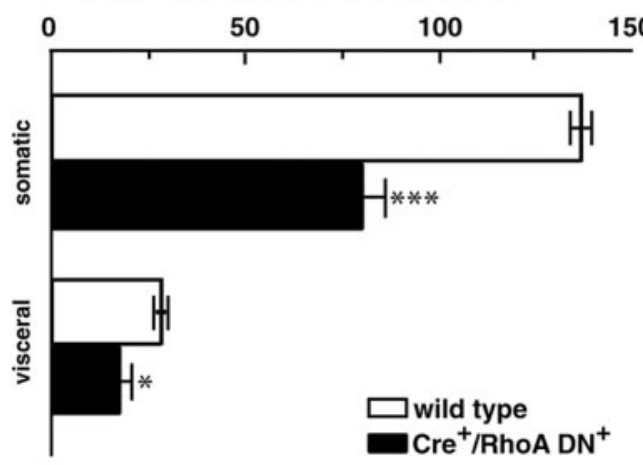

intensity of signals indicating phosphorylated MBS in the double-transgenic embryos was significantly reduced compared with the corresponding wild-type values; and reductions were $31 \%$ in $\mathrm{Cre}^{+} / \mathrm{RhoA}$ $\mathrm{DN}^{+}$embryos $(n=4 ; p<0.001)$ and $21 \%$ in $\mathrm{Cre}^{+} / \mathrm{Rho}^{-\mathrm{K} \mathrm{DN}^{+}}$embryos $(n=4 ; p<$ $0.01)$. These data indicate the reduced phosphorylation level of MBS in developing MNs in the two kinds of transgenic embryos, suggesting that the expression of RhoA DN or Rho-K DN indeed suppressed the Rho/Rho-kinase signaling pathway in these neurons.

\section{Expression of RhoA DN increases} apoptosis in the developing spinal cord We initially analyzed MN development in the $\mathrm{Cre}^{+} / \mathrm{RhoA} \mathrm{DN}^{+}$transgenic mice. To examine the generation of MNs, we used anti-Isl1 $\mathrm{Ab}$ to immunostain sections made through the spinal cord of mouse embryos at E10.5 and E12.5. Representative results of immunostaining of the wildtype and $\mathrm{Cre}^{+} / \mathrm{RhoA} \mathrm{DN}^{+}$embryos are shown in Fig. $2 \mathrm{~A}$. At E10.5, the number of Isl1-positive neurons in the transgenic embryos was markedly reduced compared with the corresponding value of the wildtype embryos, and reductions were $56 \%$ at the top cervical level, $51 \%$ at the brachial level, $48 \%$ at the thoracic level, and $42 \%$ at the lumbar level $(n=4)$ (Fig. $2 B$ ). At E12.5, somatic and visceral MN subsets at the thoracic level were distinguishable according to their positions in sections stained with Isl1 (Fig. 2A). In the transgenic embryos, the number of both somatic and visceral MNs decreased to 58 and $65 \%$ of the respective value of the wild-type embryos $(n=4)$ (Fig. $2 B$ ). To

$\mathrm{Cre}^{-} / \mathrm{Rho}-\mathrm{K} \mathrm{DN}^{+}$embryos, indicating expression of the dominant-negative forms in the double-transgenic embryos.

Activation of Rho and Rho-kinase is known to facilitate phosphorylation of MBS of myosin phosphatase (Kawano et al., 1999). To assess whether expression of RhoA DN or Rho-K DN in double-transgenic embryos could suppress the signaling pathway of Rho/Rho-kinase in developing MNs, we examined the phosphorylation level of MBS by using quantitative immunohistochemistry with antiphosphorylated MBS (Thr-850) Ab. Transverse sections through the spinal cord were prepared from wildtype and double-transgenic embryos at E10.5 and immunostained with anti-Isl1 Ab and either anti-MBS or antiphosphorylated MBS Ab. The fluorescence intensity of MBS or phosphorylated MBS signals in single Isl1-positive cells was measured, and the relative fluorescence intensity was calculated as the ratio of the fluorescence intensity in each cell relative to the average of the intensity in Isl1-positive cells of the wild-type embryos (Table 1). The relative fluorescence intensity of MBS signals in

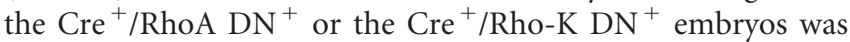
similar to the corresponding values of the wild-type embryos, indicating that the MBS protein level was not altered in the double-transgenic embryos. In contrast, the relative fluorescence determine whether the reduction in $\mathrm{MN}$ number in the $\mathrm{Cre}^{+}$/ RhoA DN ${ }^{+}$embryos was caused by apoptotic cell death, we performed TUNEL staining on E10.5 embryos (Fig. 2C). Many TUNEL-positive cells were observed in the ventral region of the spinal cord at the four different anteroposterior levels in the transgenic embryos. In contrast, TUNEL-positive cells were scarce in the corresponding regions in the wild-type embryos. Therefore, the reduced number of MNs in the transgenics is most likely attributable to increased apoptosis.

To characterize the patterning of $\mathrm{MN}$ axons projecting from the spinal cord in the Cre ${ }^{+} / \mathrm{RhoA} \mathrm{DN}^{+}$embryos, we performed anterograde and retrograde DiI labeling on E10.5 and E12.5 embryos. DiI solution was injected into the ventral region of the brachial spinal cord for anterograde labeling and into the forelimb for retrograde labeling of E10.5 embryos, whereas the solution was injected into the forelimb and the myotome for retrograde labeling of E12.5 embryos. In the wild-type embryos at E10.5, the spinal motor axons extended ventrally and reached around the base of the limb; at E12.5, these axons innervated the limb musculature and the myotome (Fig. $3 A$ ). In the transgenic embryos, motor axons grew in a similar manner to those in the wild-type embryos, although at E12.5, the axon bundles appeared 
to be slightly defasciculated, possibly because of the reduced number of MNs (Fig. $3 B$ ). The gross patterning of spinal $\mathrm{MN}$ axons thus appeared to be normal in the transgenic embryos.

The results obtained from analysis of $\mathrm{MN}$ development in the $\mathrm{Cre}^{+} / \mathrm{RhoA} \mathrm{DN}^{+}$ embryos indicate that the expression of RhoA DN particularly induced apoptotic cell death in the ventral spinal cord while preserving the gross patterning of the spinal motor axons.

Expression of Rho-K DN increases apoptosis in the developing spinal cord MN development in the $\mathrm{Cre}^{+} / \mathrm{Rho}^{\mathrm{K}}$ $\mathrm{DN}^{+}$transgenic mice was examined by the same procedures as those used for the analysis of the $\mathrm{Cre}^{+} / \mathrm{RhoA} \mathrm{DN}^{+}$embryos. Developmental defects in the $\mathrm{Cre}^{+} / \mathrm{Rho}^{-\mathrm{K}}$ $\mathrm{DN}^{+}$embryos were very similar to those in the $\mathrm{Cre}^{+} / \mathrm{RhoA} \mathrm{DN}^{+}$embryos. The number of the spinal MNs at the thoracic level in the $\mathrm{Cre}^{+} / \mathrm{Rho}^{-\mathrm{K} \mathrm{DN}^{+}}$embryos was significantly decreased relative to the corresponding values for the wild types, and the decrease was $41 \%$ at E10.5, $26 \%$ in somatic MNs, and $33 \%$ in visceral MNs at E12.5 $(n=4)$ (Fig. $4 A$ ). The diminished MN number in the transgenic embryos was derived from increased apoptosis in the ventral region of the spinal cord at the thoracic level (Fig. $4 B)$. The increased apoptosis was also observed in the top cervical, brachial, and lumbar levels of the transgenic embryos (data not shown). The gross patterning of the spinal motor axons in the $\mathrm{Cre}^{+} / \mathrm{Rho}-\mathrm{K}$ $\mathrm{DN}^{+}$embryo appeared to be unaffected (Fig. 4C). The similarity of developmental defects between the $\mathrm{Cre}^{+} / \mathrm{RhoA} \mathrm{DN}^{+}$and $\mathrm{Cre}^{+} / \mathrm{Rho}^{\mathrm{K} \mathrm{DN}}{ }^{+}$embryos suggests that the Rho-dependent actions observed in $\mathrm{MN}$ development were predominantly mediated by Rho-kinase.

\section{Increased apoptosis of MNs at early embryonic stages in transgenic mice}

In the normal development of mice, PCD of MNs occurs in the spinal cord at E12.5-E16.5 (Lance-Jones, 1982; Yamamoto and Henderson, 1999). We examined the time course of apoptotic cell death in the double-transgenic embryos from E9.5 to E11.5 by using Isl1 immunostaining combined with TUNEL staining. We used the $\mathrm{Cre}^{+} / \mathrm{Rho}^{\mathrm{K} \mathrm{DN}^{+}}$embryos to examine the effects on apoptosis of the suppression of Rho signaling pathway through Rho-kinase. As shown in Figure $5 A$, a few apoptotic signals appeared in the developing MNs at E9.5, and the number of signals gradually increased until E10.5 and then decreased at E11.5. Confocal images of the double staining of E10.5 embryos revealed the presence of TUNEL-positive signals in Isl1-positive MNs (20$25 \% ; n=4)$. In addition, we examined the activation of caspase- 3 in the transgenic embryos at E10.5 by using immunostaining for activated caspase- 3 combined with Isll staining (Fig. 5B). Confocal images of the double-stained cells revealed signals for activated caspase-3 in some MNs. Moreover, we analyzed the mor- phology of apoptotic cells in the embryos by using electron microscopy (Fig. 5C). In the transgenic embryos, condensation of chromatin structure, electron-dense cytoplasm, and shrinkage of the soma in degenerating neurons were seen, and signs of phagocytosis of dying cells were also observed. These data show that in the transgenic mice, MNs did differentiate but underwent apoptosis at the early embryonic stages before the initiation of PCD.

Cell death of MNs at late embryonic stages in transgenic mice To examine the influence of suppression of Rho/Rho-kinase signaling on $\mathrm{MN}$ death at the late embryonic stages corresponding to the period of PCD, we performed Isl1 immunostaining combined with TUNEL staining on E14.5 and E16.5 embryos. As shown in Figure $6 A$, at E14.5, a few TUNEL-positive signals were observed in the somatic MN column in the wild-type embryos, whereas the number of the signals seemed to be moderately increased in the $\mathrm{Cre}^{+} / \mathrm{Rho}^{-\mathrm{K} \mathrm{DN}^{+}}$transgenic embryos. At E16.5, there were no TUNEL-positive signals in either of the two kinds of embryos. In addition, the number of Isl1-positive cells in the wild-type embryos was reduced along with the progress of development, and the number of cells in the transgenic embryos was 
A

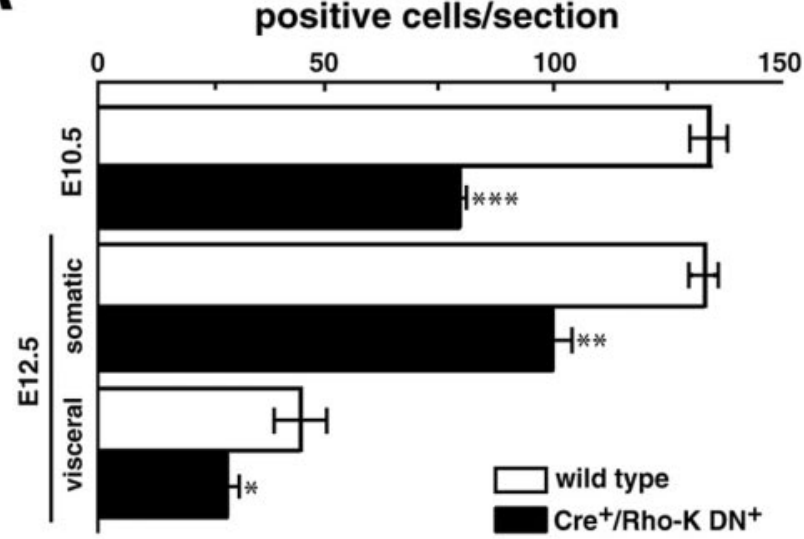

B
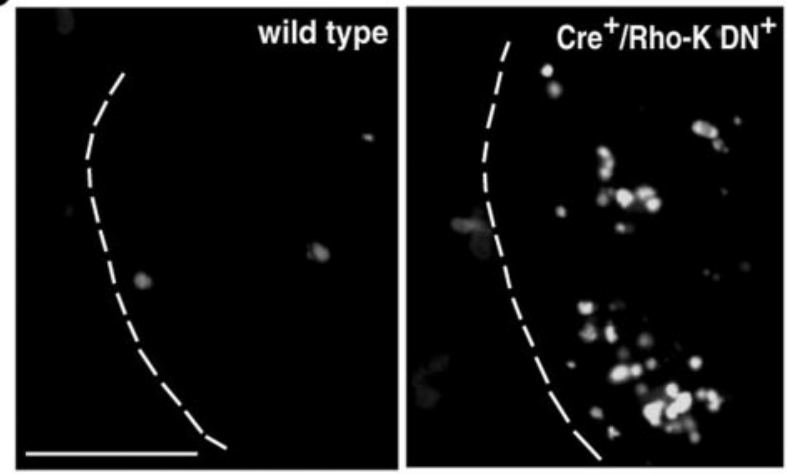

C
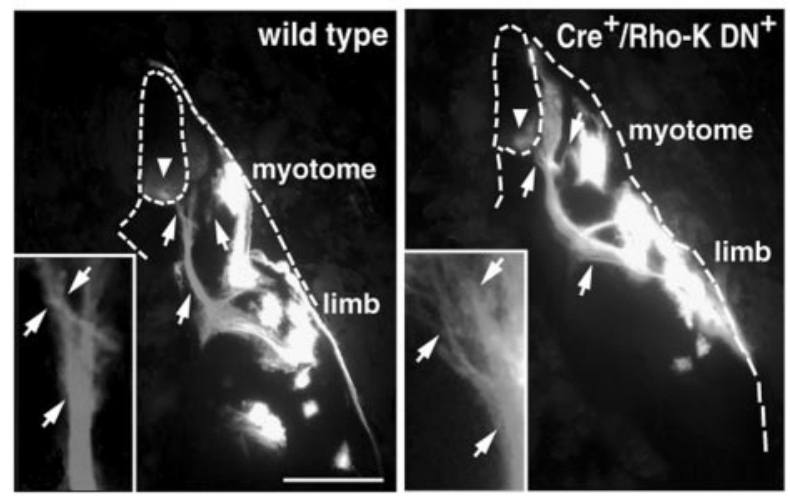

Figure 4. MN development and axon patterning in Cre ${ }^{+} / \mathrm{Rho}_{-} \mathrm{KDN}^{+}$mice. $A$, Cell number of MNs in the thoracic spinal cord. Data are the mean \pm SEM (20-30 sections from 4 embryos). Asterisks indicate $p$ values according to Student's $t$ test; ${ }^{*} p<0.05,{ }^{* *} p<0.01,{ }^{* * *} p<0.001$ versus wild-type mice. $B$, TUNEL staining of sections through the thoracic region of transgenic embryo at E10.5. Scale bar, $50 \mu \mathrm{m}$. C, Retrograde labeling of spinal motor axons of E12.5 embryos. Dil solution was injected into the forelimb and myotome for labeling. Arrows and arrowheads indicate labeled axons and cell bodies, respectively. Insets show higher magnification of axons projecting ventrally from the proximal ventral neural tube. Scale bar, $500 \mu \mathrm{m}$.

fewer than that for the corresponding values of the wild types (Fig. $6 B$ ). When the ratio of the number relative to the average of the wild-type values at each stage was calculated, the ratio in the transgenic embryos at E16.5 (0.68 \pm 0.01$)$ was slightly but significantly reduced relative to that at E14.5 $(0.73 \pm 0.02)(p<0.05$; Student's $t$ test). In the transgenic mice, $\mathrm{MN}$ death thus appeared to be moderately enhanced at the late embryonic stages.
Apparently normal development of peripheral tissues in transgenic embryos

Synaptic target tissues such as the limb musculature and the myotome are known to provide trophic support for the survival of developing MNs (Oppenheim, 1991). Also, Schwann cells, which surround the growing axons, are sources of trophic support for $\mathrm{MN}$ survival. If these structures would be damaged in our transgenic embryos, this damage may have secondarily caused the increase in cell death of MNs. To test this possibility, we explored the morphogenesis of the peripheral tissues in the $\mathrm{Cre}^{+} / \mathrm{Rho}^{-\mathrm{K}}$ $\mathrm{DN}^{+}$embryos at E16.5. Staining with hematoxylin and eosin of transverse sections through the limb showed that the gross morphology of the tissues, including the muscle, was unaffected in the transgenic embryos (Fig. 7A). Immunostaining for S100, which is a marker of developing Schwann cells, combined with neurofilament staining indicated normal development of Schwann cells around nerve fibers in these embryos (Fig. $7 B$ ). In addition, the histological staining of $\beta$-gal activity in $\mathrm{Cre}^{+} / \mathrm{lac}^{+}$embryos exhibited no Cre-loxP recombination signals in these structures (data not shown). These data thus suggest that the increase in death of MNs in transgenic mice is predominantly attributable to the suppression of the Rho/Rho-kinase signaling in these neurons per se.

\section{Discussion}

In the present study, we investigated the role of the Rho/Rhokinase signaling pathway in the development of MNs in the spinal cord. For suppression of the activity of Rho and Rho-kinase in the developing MNs, the RhoA DN or Rho-K DN gene was expressed in these neurons under the control of the Cre-loxP recombination system in transgenic mice. Expression of RhoA DN reduced the number of MNs because of increased apoptotic cell death in the ventral spinal cord while preserving the gross patterning of spinal motor axons. Expression of Rho-K DN produced developmental defects resembling those observed consequential to the RhoA DN expression. In addition, the analysis of transgenic mice expressing Rho-K DN showed that the increased apoptosis of MNs was induced at the early embryonic stages before the initiation of PCD, and that MN death at the late embryonic stages corresponding to the period of PCD was moderately enhanced in the transgenic embryos. However, the Rho-K DN expression did not affect the development of the peripheral tissues including the muscle and Schwann cells. Together, these data indicate that the Rho signaling pathway, primarily through Rho-kinase, plays a crucial role in the survival of spinal MNs during embryonic development.

Our findings of the increased apoptosis of MNs before the onset of PCD in the transgenic mice provide evidence that the Rho/Rho-kinase signaling facilitates the survival of developing MNs at the early embryonic stages (E9.5-E11.5). The axonal labeling experiments showed that spinal motor axons did not innervate the final synaptic targets, including the limb musculature and the myotome, at such early stages (E10.5). These data implicate the Rho/Rho-kinase pathway in the mechanism for the survival, at least independently of trophic support derived from these synaptic targets. Recent evidence suggests the presence of mechanisms that regulate neuronal survival or death in a synaptic target-independent manner (Oppenheim et al., 2001). Indeed, growing axons are electrically active before innervating the targets (Milner and Landmesser, 1999; Hanson and Landmesser, 2003). One possible mechanism by which the Rho/Rho-kinase pathway mediates MN survival during early development is dependent on local trophic factors provided from the peripheral 
tissues interacting with growing axons. Hepatocyte growth factor-scatter factor (HGF-SF) seems to function as a neurotrophic factor originating from the sclerotome and limb mesenchyme for the spinal MNs (Ebens et al., 1996). The Rho/ Rho-kinase pathway is reported to be necessary for cell motility induced by HGF-SF in cultured epithelial cells (Fukata et al., 1999). These data suggest that this pathway may mediate the survival of developing MNs in response to HGF-SF provided from some peripheral tissues as intermediate targets. Another possible mechanism involved in the prevention of apoptotic $\mathrm{MN}$ death is the regulation of cell adhesion. The Rho/Rho-kinase pathway is reported to control integrinmediated cell-matrix interactions (Howe et al., 1998). Inadequate interaction between cell and matrix is known to cause cytoskeletal perturbation, thereby leading to apoptosis (Frisch and Screaton, 2001). The data suggest that this pathway may act to prevent developing MNs from apoptosis downstream of integrin-mediated cellmatrix adhesion.

We observed moderately enhanced $\mathrm{MN}$ death in transgenic mice at the late embryonic stages (E14.5-E16.5) corresponding to a period of PCD. Survival or death of MNs during PCD is generally considered to depend on limiting amounts of neurotrophic factors derived from their synaptic targets (Oppenheim, 1991; Pettmann and Henderson, 1998; Sendtner et al., 2000; Huang and Reichardt, 2001). Rho is known to bind a member of the family of receptors for nerve growth factor to regulate axonal growth in retinal neurons (Yamashita et al., 1999). Again, Rho is reported to mediate axonal responses triggered by nerve growth factor, neurotrophin-3, and brainderived neurotrophic factor in cultured cell lines (Özdinler and Erzurumlu, 2001). These data suggest that the Rho/Rho-kinase signaling may participate in the survival of developing MNs downstream of these neurotrophins during the time of PCD.

MNs are generated from a specific progenitor domain in the ventral neural tube, and these cells acquire their specific cell fate as they become postmitotic (Ericson et al., 1997). Our analysis of Cre-loxP recombination pattern in the $\mathrm{Cre}^{+} / \mathrm{lacZ}^{+}$transgenic embryos indicated that the recombination occurred in the developing $\mathrm{MNs}$ and in their presumptive progenitor cells. Analysis of the time course of cell death in the Cre ${ }^{+} / \mathrm{Rh}-\mathrm{K} \mathrm{DN}^{+}$embryos revealed that $\mathrm{MNs}$ acquired their cell fate and then underwent apoptosis. These data suggest that suppression of the Rho/Rho-kinase signaling does not appear to alter specification of the cell fate of MNs. In addition, previous studies on neuroblastoma cells show

C versus wild-type mice.
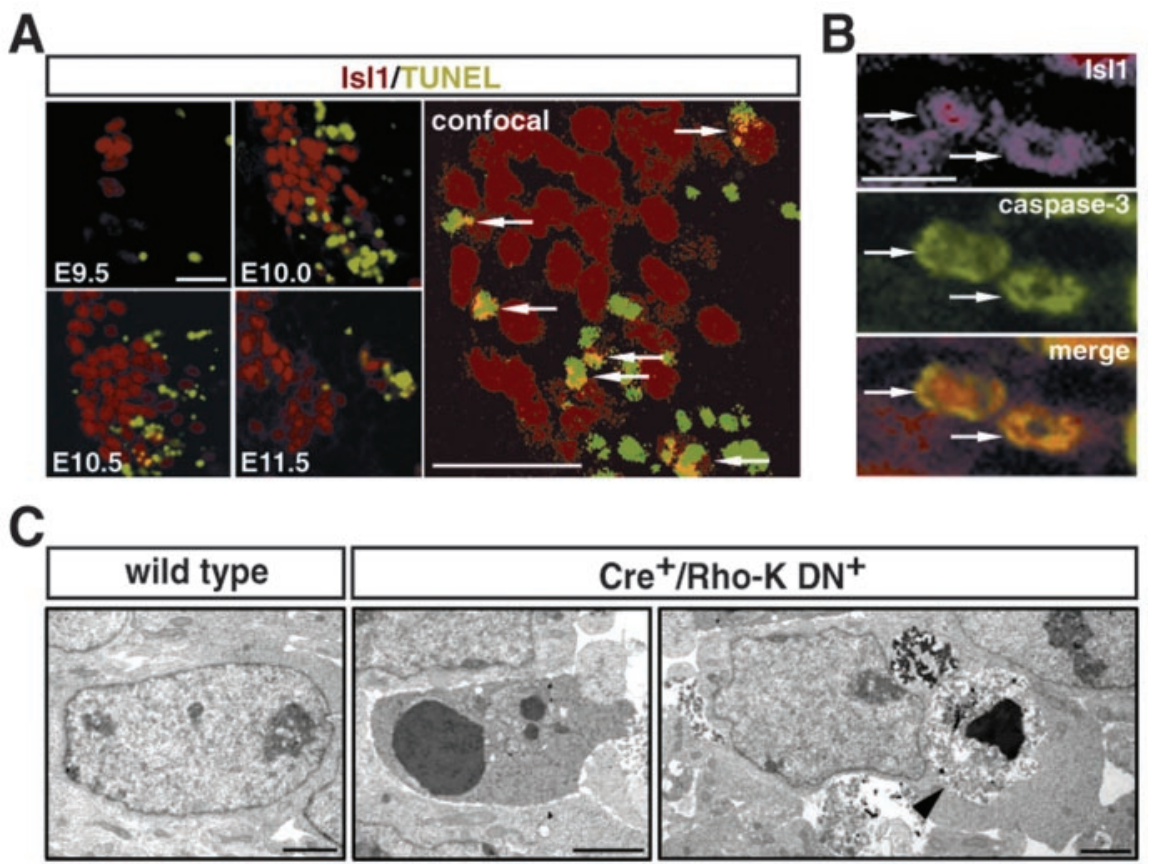

Figure 5. MN apoptosis during early embryonic development in $\mathrm{Cre}^{+} / \mathrm{Rho}_{\mathrm{K}} \mathrm{K} \mathrm{DN}^{+}$mice. $A$, Time course of apoptotic cell death. Sections through the spinal cord at the thoracic level were prepared from transgenic embryos at various developmental stages and analyzed by $\mathrm{Is} \mid 1 \mathrm{immunostaining} \mathrm{combined} \mathrm{with} \mathrm{TUNEL} \mathrm{staining.} \mathrm{A} \mathrm{confocal} \mathrm{image} \mathrm{of} \mathrm{the} \mathrm{section} \mathrm{from} \mathrm{an} \mathrm{E10.5} \mathrm{embryo}$ shows TUNEL-positive signals present in degenerating MNs (arrows). Scale bar, $25 \mu \mathrm{m}$. B, Activation of caspase-3. Sections through the thoracic spinal cord of E10.5 transgenic embryos were analyzed by IsI1 immunostaining combined with staining for activated caspase-3. A confocal image of the section shows activated caspase-3 in degenerating cells (arrows). Scale bar, $10 \mu \mathrm{m}$. C, Morphology of apoptotic cells. Ultrathin sections through the thoracic spinal cord of an E10.5 embryo were stained. Representative electron micrographs of cells in the MN column from the wild-type and transgenic embryos are shown. In the transgenics, condensation of chromatin, dark cytoplasm, and shrinkage of the soma in degenerating neurons are evident. Arrowhead points to a dying cell engulfed by a phagocytic cell. Scale bar, $2 \mu \mathrm{m}$.

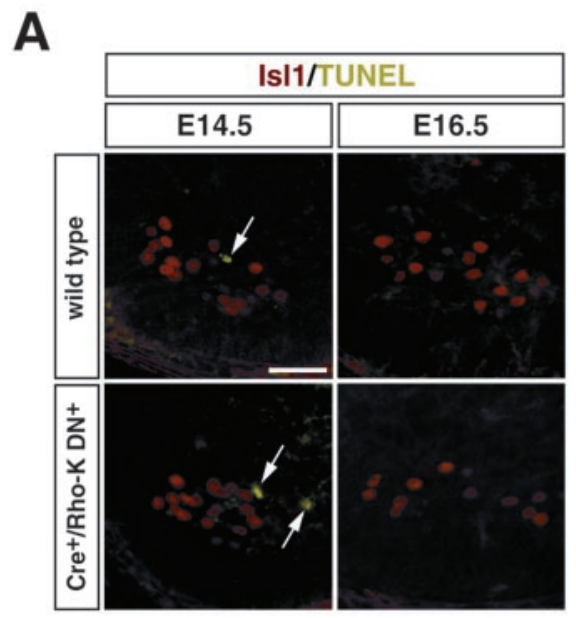

B

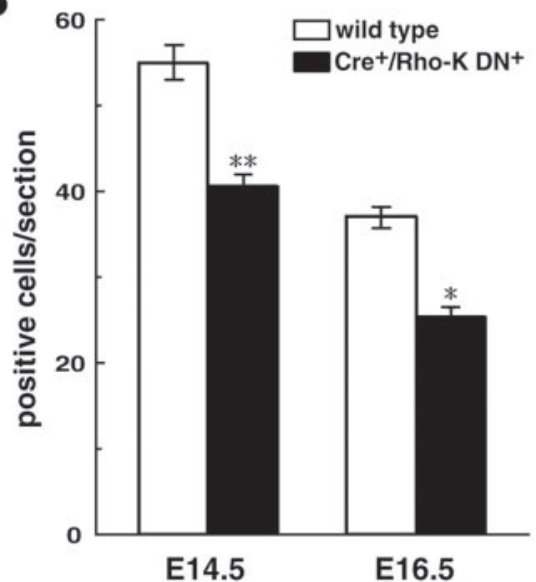

Figure 6. MN apoptosis during late embryonic development in Cre ${ }^{+} / \mathrm{Rho-K} \mathrm{DN}{ }^{+}$mice. $A$, Isl1 immunostaining combined with TUNEL staining. Sections through the thoracic spinal cord of transgenic embryos at E14.5 and E16.5 were used for double immunostaining. Arrows point to TUNEL signals in the MN columns. Scale bar, $50 \mu \mathrm{m}$. $B$, Cell number of MNs. Data are the mean \pm SEM ( $20-30$ sections from 4 embryos). Asterisks indicate $p$ values according to Student's $t$ test; ${ }^{*} p<0.01,{ }^{* *} p<0.001$

that the morphology of growth cones is regulated through the Rho/Rho-kinase signaling (Amano et al., 1998; Hirose et al., 1998; Luo, 2000). In the present study, the gross patterning of spinal motor axons in the transgenic embryos was apparently normal, thus suggesting that the regulation of growth cone morphology through the Rho/Rho-kinase signaling may not be re- 
A

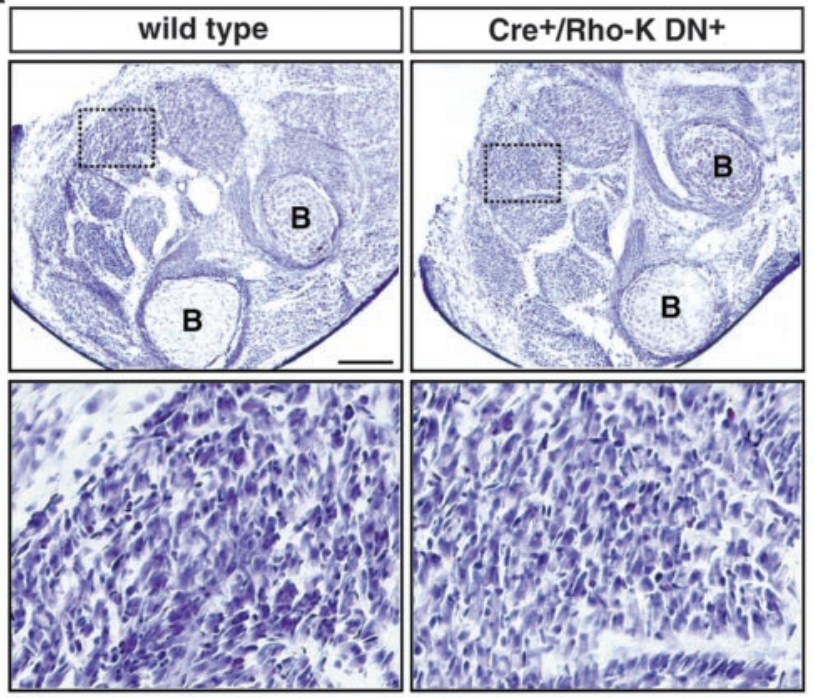

B

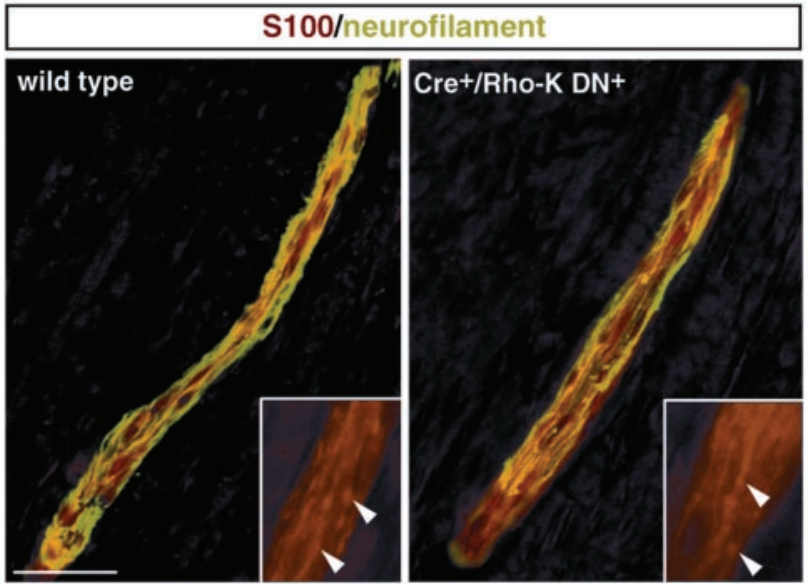

Figure 7. Morphology of the peripheral tissues in $\mathrm{Cre}^{+} / \mathrm{Rho}_{0} \mathrm{~K} \mathrm{DN}{ }^{+}$mice. A, Hematoxylineosin staining of transverse sections through the forelimb. Bottom panels show 25 -foldmagnified views of the skeletal musculature (dotted boxes in the top panels). B, Bone. Scale bar, $200 \mu \mathrm{m} . B$, Immunostaining for $\mathrm{S} 100$ and neurofilament of the sections through the thoracic spinal cord. Insets show higher magnification of S100-positive Schwann cells on growing axons (arrowheads). Scale bar, $50 \mu \mathrm{m}$.

quired for gross axon patterning of spinal MNs. However, our axonal labeling experiments did not distinguish motor axons derived from discrete subpopulations of MNs, which are located in different parts of the MN column and project to different regions in the limb (Goulding, 1998; Lin et al., 1998; Shirasaki and Paff, 2002). It remains to be determined whether there are any subtle changes in axonal projections from each $\mathrm{MN}$ subpopulation in our transgenic mice.

We observed the Cre-loxP recombination under the control of the human DBH gene promoter in SG neurons and in some of the DRG neurons in addition to MNs during embryonic development. Our preliminary analysis of the development of SG and DRG neurons in the Cre ${ }^{+} / \mathrm{Rho}^{-} \mathrm{K} \mathrm{DN}^{+}$transgenic mice showed that the morphogenesis of these ganglia and the number of neurons were apparently unaltered at the early and late embryonic stages (see supplementary Fig. 1; available at www.jneurosci.org). Therefore, Rho/Rho-kinase signaling does not seem to be essential for the survival of SG and DRG neurons during embryogen- esis. Recent evidence shows that other small GTPases, such as Ras and Rap1, transmit signals for the survival of these neurons (Klesse and Parada, 1998; Mazzoni et al., 1999; York et al., 2000).

Finally, we developed the Cre-loxP recombination system in specific cell types by using the $\mathrm{DBH}$ gene promoter. This Cre-loxP system enabled us to elucidate the in vivo role of the Rho/Rhokinase signaling pathway in the development of particular neuronal types. This system will provide a useful experimental tool for studying the functions of other gene products in animal development, physiology, and pathology that are expressed in the neuronal types of interest.

\section{References}

Amano M, Chihara K, Kimura K, Fukata Y, Nakamura N, Matsuura Y, Kaibuchi K (1997) Formation of actin stress fibers and focal adhesions enhanced by Rho-kinase. Science 275:1308-1311.

Amano M, Chihara K, Nakamura N, Fukata Y, Yano T, Shibata M, Ikebe M, Kaibuchi K (1998) Myosin II activation promotes neurite retraction during the action of Rho and Rho-kinase. Genes Cells 3:177-188.

Araki K, Araki M, Miyazaki J, Vassalli P (1995) Site-specific recombination of a transgene in fertilized eggs by transient expression of Cre recombinase. Proc Natl Acad Sci USA 92:160-164.

Aznar S, Lacal JC (2001) Rho signals to cell growth and apoptosis. Cancer Lett 165:1-10.

Barrett K, Leptin M, Settleman J (1997) The Rho GTPase and a putative RhoGEF mediate a signaling pathway for the cell shape changes in Drosophila gastrulation. Cell 91:905-915.

Ebens A, Brose K, Leonardo ED, Hanson Jr MG, Bladt F, Birchmeier C, Barres BA, Tessier-Lavigne M (1996) Hepatocyte growth factor/scatter factor is an axonal chemoattractant and a neurotrophic factor for spinal motor neurons. Neuron 17:1157-1172.

Ericson J, Rashbass P, Schedl A, Brenner-Morton S, Kawakami A, van Heyningen V, Jessell TM, Briscoe J (1997) Pax6 controls progenitor cell identity and neuronal fate in response to graded Shh signaling. Cell 90:169-180.

Frisch SM, Screaton RA (2001) Anoikis mechanisms. Curr Opin Cell Biol 13:555-562.

Fukata Y, Oshiro N, Kinoshita N, Kawano Y, Matsuoka Y, Bennett V, Matsuura Y, Kaibuchi K (1999) Phosphorylation of adducin by Rho-kinase plays a crucial role in cell motility. J Cell Biol 145:347-361.

Goulding M (1998) Specifying motor neurons and their connections. Neuron 21:943-946.

Gu H, Zou YR, Rajewsky K (1993) Independent control of immunoglobulin switch recombination at individual switch regions evidenced through Cre-loxP-mediated gene targeting. Cell 73:1155-1164.

Hall A (1998) Rho GTPases and the actin cytoskeleton. Science 279:509-514.

Hanson MG, Landmesser LT (2003) Characterization of the circuits that generate spontaneous episodes of activity in the early embryonic mouse spinal cord. J Neurosci 23:587-600.

Hirose M, Ishizaki T, Watanabe N, Uehata M, Kranenburg O, Moolenaar WH, Matsumura F, Maekawa M, Bito H, Narumiya S (1998) Molecular dissection of the Rho-associated protein kinase (p160ROCK)-regulated neurite remodeling in neuroblastoma N1E-115 cells. J Cell Biol 141:1625-1636.

Homma S, Yaginuma H, Oppenheim RW (1994) Programmed cell death during the earliest stages of spinal cord development in the chick embryo: a possible means of early phenotypic selection. J Comp Neurol 345:377-395.

Howe A, Aplin AE, Alahari SK, Juliano RL (1998) Integrin signaling and cell growth control. Curr Opin Cell Biol 10:220-231.

Huang EJ, Reichardt LF (2001) Neurotrophins: roles in neuronal development and function. Annu Rev Neurosci 24:677-736.

Ishizaki T, Maekawa M, Fujisawa K, Okawa K, Iwamatsu A, Fujita A, Watanabe N, Saito Y, Kakizuka A, Morii N, Narumiya S (1996) The small GTP-binding protein Rho binds to and activates a $160 \mathrm{kDa}$ Ser/Thr protein kinase homologous to myotonic dystrophy kinase. EMBO J 15:1885-1893.

Kaibuchi K, Kuroda S, Amano M (1999) Regulation of the cytoskeleton and cell adhesion by the Rho family GTPases in mammalian cells. Annu Rev Biochem 68:459-486. 
Kapur RP, Hoyle GW, Mercer EH, Brinster RL, Palmiter RD (1991) Some neuronal cell populations express human dopamine $\beta$-hydroxylase-lac $Z$ transgenes transiently during embryonic development. Neuron 7:717-727.

Kawano Y, Fukata Y, Oshiro N, Amano M, Nakamura T, Ito M, Matsumura F, Inagaki M, Kaibuchi K (1999) Phosphorylation of myosin-binding subunit (MBS) of myosin phosphatase by Rho-kinase in vivo. J Cell Biol 147:1023-1037.

Klesse LJ, Parada LF (1998) p21 ras and phosphatidylinositol-3 kinase are required for survival of wild-type and NF1 mutant sensory neurons. J Neurosci 18:10420-10428.

Kobayashi K, Sasaoka T, Morita S, Nagatsu I, Iguchi A, Kurosawa Y, Fujita K, Nomura T, Kimura M, Katsuki M, Nagatsu T (1992) Genetic alteration of catecholamine specificity in transgenic mice. Proc Natl Acad Sci USA 89:1631-1635.

Lance-Jones C (1982) Motoneuron cell death in the developing lumbar spinal cord of the mouse. Brain Res 256:473-479.

Leung T, Manser E, Tan L, Lim L (1995) A novel serine/threonine kinase binding the Ras-related RhoA GTPase which translocates the kinase to peripheral membranes. J Biol Chem 270:29051-29054.

Lin JH, Saito T, Anderson DJ, Lance-Jones C, Jessell TM, Arber S (1998) Functionally related motor neuron pool and muscle sensory afferent subtypes defined by coordinate ETS gene expression. Cell 95:393-407.

Luo L (2000) Rho GTPases in neuronal morphogenesis. Nat Rev Neurosci 1:173-180.

Magie CR, Meyer MR, Gorsuch MS, Parkhurst SM (1999) Mutations in the Rhol small GTPase disrupt morphogenesis and segmentation during early Drosophila development. Development 126:5353-5364.

Matsui T, Amano M, Yamamoto T, Chihara K, Nakafuku M, Ito M, Nakano T, Okawa K, Iwamatsu A, Kaibuchi K (1996) Rho-associated kinase, a novel serine/threonine kinase, as a putative target for small GTP binding protein Rho. EMBO J 15:2208-2216.

Matsushita N, Okada H, Yasoshima Y, Takahashi K, Kiuchi K, Kobayashi K (2002) Dynamics of tyrosine hydroxylase promoter activity during midbrain dopaminergic neuron development. J Neurochem 82:295-304.

Mazzoni IE, Said FA, Aloyz R, Miller FD, Kaplan D (1999) Ras regulates sympathetic neuron survival by suppressing the p53-mediated cell death pathway. J Neurosci 19:9716-9727.

Mercer EH, Hoyle GW, Kapur RP, Brinster RL, Palmiter RD (1991) The dopamine $\beta$-hydroxylase gene promoter directs expression of E. coli lacZ to sympathetic and other neurons in adult transgenic mice. Neuron 7:703-716.

Mettling C, Gouin A, Robinson M, el M'Hamdi H, Camu W, Bloch-Gallego E, Buisson B, Tanaka H, Davies AM, Henderson CE (1995) Survival of newly postmitotic motoneurons is transiently independent of exogenous trophic support. J Neurosci 15:3128-3137.

Milner LD, Landmesser LT (1999) Cholinergic and GABAergic inputs drive patterned spontaneous motoneuron activity before target contact. J Neurosci 19:3007-3022.
Oppenheim RW (1991) Cell death during development of the nervous system. Annu Rev Neurosci 14:453-501.

Oppenheim RW, Calderó J, Esquerda J, Gould TW (2001) Targetindependent programmed cell death in the developing nervous system. In: Handbook of brain and behaviour in human development (Kalverboer AF, Gramsbergen A, eds), pp 343-407. Groningen: Kluwer.

Özdinler PH, Erzurumlu RS (2001) Regulation of neurotrophin-induced axonal responses via Rho GTPases. J Comp Neurol 438:377-387.

Pettmann B, Henderson CE (1998) Neuronal cell death. Neuron 20:633-647.

Sasaoka T, Kobayashi K, Nagatsu I, Takahashi R, Kimura M, Yokoyama M, Nomura T, Katsuki M, Nagatsu T (1992) Analysis of the human tyrosine hydroxylase promoter-chloramphenicol acetyltransferase chimeric gene expression in transgenic mice. Mol Brain Res 16:274-286.

Sendtner M, Pei G, Beck M, Schweizer U, Wiese S (2000) Developmental motoneuron cell death and neurotrophic factors. Cell Tissue Res 301:71-84.

Shirasaki R, Pfaff SL (2002) Transcriptional codes and the control of neuronal identity. Annu Rev Neurosci 25:251-281.

Strutt DI, Weber U, Mlodzik M (1997) The role of RhoA in tissue polarity and Frizzled signalling. Nature 387:292-295.

Takahashi M, Osumi N (2002) Pax6 regulates specification of ventral neurone subtypes in the hindbrain by establishing progenitor domains. Development 129:1327-1338.

Wei L, Roberts W, Wang L, Yamada M, Zhang S, Zhao Z, Rivkees SA, Schwartz RJ, Imanaka-Yoshida K (2001) Rho kinases play an obligatory role in vertebrate embryonic organogenesis. Development 128:2953-2962.

Winter CG, Wang B, Ballew A, Royou A, Karess R, Axelrod JD, Luo L (2001) Drosophila Rho-associated kinase (Drok) links Frizzled-mediated planar cell polarity signaling to the actin cytoskeleton. Cell 105:81-91.

Wünnenberg-Stapleton K, Blitz IL, Hashimoto C, Cho KW (1999) Involvement of the small GTPases XRhoA and XRnd1 in cell adhesion and head formation in early Xenopus development. Development 126:5339-5351.

Yaginuma H, Tomita M, Takashita N, McKay SE, Cardwell C, Yin QW, Oppenheim RW (1996) A novel type of programmed neuronal death in the cervical spinal cord of the chick embryo. J Neurosci 16:3685-3703.

Yamamoto Y, Henderson CE (1999) Patterns of programmed cell death in populations of developing spinal motoneurons in chicken, mouse, and rat. Dev Biol 214:60-71.

Yamashita T, Tucker KL, Barde YA (1999) Neurotrophin binding to the p75 receptor modulates Rho activity and axonal outgrowth. Neuron 24:585-593.

York RD, Molliver DC, Grewal SS, Stenberg PE, McCleskey EW, Stork PJ (2000) Role of phosphoinositide 3-kinase and endocytosis in nerve growth factor-induced extracellular signal-regulated kinase activation via Ras and Rap1. Mol Cell Biol 20:8069-8083. 